\title{
Exploration of radiation-induced lung injury, from mechanism to treatment: a narrative review
}

\author{
Yujie Yan ${ }^{1}$, Jiamei Fu ${ }^{1}$, Roman O. Kowalchuk ${ }^{2}$, Christopher M. Wright ${ }^{3}$, Ran Zhang ${ }^{1}$, Xuefei Li ${ }^{4}$, Yaping Xu ${ }^{1}$ \\ ${ }^{1}$ Department of Radiation Oncology, Shanghai Pulmonary Hospital, Tongji University Medical School Cancer Institute, Tongji University School \\ of Medicine, Shanghai, China; ${ }^{2}$ Department of Radiation Oncology, Mayo Clinic, Rochester, MN, USA; ${ }^{3}$ Department of Radiation Oncology, \\ University of Pennsylvania, Philadelphia, PA, USA; ${ }^{4}$ Department of Lung Cancer and Immunology, Shanghai Pulmonary Hospital \& Thoracic \\ Cancer Institute, Tongji University School of Medicine, Shanghai, China \\ Contributions: (I) Conception and design: Y Yan; (II) Administrative support: Y Xu; (III) Provision of study materials or patients: R Zhang; (IV) \\ Collection and assembly of data: J Fu; (V) Data analysis and interpretation: Y Yan; (VI) Manuscript writing: All authors; (VII) Final approval of \\ manuscript: All authors. \\ Correspondence to: Yaping Xu, MD. Department of Radiation Oncology, Shanghai Pulmonary Hospital, Tongji University School of Medicine, \\ Shanghai 200433, China. Email: xuyaping1207@163.com; Xuefei Li, MD. Department of Lung Cancer and Immunology, Shanghai Pulmonary \\ Hospital \& Thoracic Cancer Institute, Tongji University School of Medicine, Shanghai 200433, China. Email: bug_lily2003@163.com; Ran \\ Zhang, MD. Department of Radiation Oncology, Shanghai Pulmonary Hospital, Tongji University School of Medicine, Shanghai 200433, China. \\ Email: zhangranwork@foxmail.com.
}

Background and Objective: Radiation-induced lung injury (RILI) is often found in thoracic tumor patients after thoracic radiation therapy, and influences patient quality of life. However, systematic exploration of RILI, including its molecular biological mechanisms and standardized treatment, has not yet been fully elucidated. The main objective of the narrative review was to describe the available evidence concerning RILI, from the biological mechanism to the clinical management. The underlying causes of RILI are multifactorial, including gene-level changes, the influence of signaling pathways, the convergence of various cells, as well as the expression of cytokines and chemokines. Based on the various mechanisms of RILI, several novel treatment strategies have been proposed and gradually applied in clinical practice.

Methods: PubMed was used to collect articles about RILI from 1995 to 2021. The papers included clinical trials, reviews, as well as systematic reviews and meta-analyses. Based on the mechanism, diagnosis, and treatment, we synthesized and analyzed these papers to form a clearly logical and normative suggestion to guide clinical application.

Key Content and Findings: RILI is a constantly developing and changing process including radiation pneumonitis and radiation lung fibrosis. Different kinds of inflammatory and immune cells such as macrophages, fibroblasts, and T cells play key roles in the development of RILI, and transforming growth factor- $\beta$ (TGF- $\beta$ ), interleukin-4 (IL-4), IL-13, and interferon- $\gamma$ (IFN- $\gamma$ ) are also participants in this process. At present, glucocorticoids are mainly therapeutic drugs for the early stage of RILI, and drugs treatment should abide early period, sufficient doses, and the individual principles. Other novel drugs such as Azithromycin also have been tried in clinical application. radiation dose, combination therapy modality, the condition of the tumor, and the age and underlying conditions of patients all effect the occurrence of RILI. Importantly, RILI has a relatively higher incidence in patients who received radiotherapy combined with other treatments, especially immunotherapy.

Conclusions: The occurrence of RILI after radiotherapy will greatly affect the prognosis and quality of life of patients. In clinical practice, early intervention, active treatment, and more effective therapeutic drugs should be found.

Keywords: Radiation-induced lung injury (RILI); mechanism; treatment; predisposing factors

Submitted Dec 07, 2021. Accepted for publication Feb 21, 2022.

doi: $10.21037 /$ tlcr-22-108

View this article at: https://dx.doi.org/10.21037/tlcr-22-108 


\section{Introduction}

Radiation therapy is an integral part of oncologic treatment for thoracic tumors. With the advancement of therapeutic technology and radiotherapy methods, the adverse effects of radiotherapy have gradually decreased. However, when ionizing radiation kills tumor cells, it also causes damage to healthy cells. We recognize the damage in lung tissue as radiation-induced lung injury (RILI), which occurs due to exposure to ionizing radiation, leading to pathophysiological and interstitial changes on imaging. As a key potential adverse event of thoracic radiotherapy, the incidence of RILI has reached as high as $30 \%$ in some settings (1).

RILI is divided into two stages: radiation pneumonia (RP) in the early stage, and radiation lung fibrosis (RLF) in the later stages. This is marked by a series of pathophysiological changes, including epithelial and endothelial cell damage, infiltration of inflammatory cells, release of cytokines, differentiation of fibroblasts, deposition of extracellular matrix (ECM), and synthesis of collagen. These processes ultimately lead to imaging and clinical changes. On the molecular level, these changes correspond to a series of non-specific symptoms including cough, weakness, dyspnea, fever, abnormal imaging, and others. Thus, research into treatments and prevention of these diseases is crucial.

Over the past few decades, an enormous amount of research based on RILI has been conducted from different angles (2-4). Different radiotherapy doses and radiomics parameters affected the development of RILI has been described in prior literature. In recent years, the advent of contemporary chemo and immune therapeutics has also shifted our understanding of the development of RILI.
Herein, we comprehensively review RILI, from mechanism to treatment, and analysis the predisposing factor of RILI. We observed that prescriptive therapy was more helpful in reducing the severity of RILI including early detection of disease, and application of glucocorticoids (GCs) or other novel drugs. Moreover, while recognizing that immunotherapy combined with radiotherapy can bring greater survival benefits to patients, we are always vigilant against the occurrence of treatment-related pneumonia (5).

The review describes the molecular and cellular mechanisms of RILI, focuses on the current clinical treatment methods for RILI and puts forward reasonable suggestions for drug treatment doses, and emphasizes the occurrence of RILI under combined immunotherapy and radiotherapy. We present the following article in accordance with the Narrative Review reporting checklist (available at https://tlcr.amegroups.com/article/view/10.21037/tlcr-22$108 / \mathrm{rc})$.

\section{Methods}

This narrative review was conducted using the PubMed website. We collected articles about RILI from 1995 to 2021. The following search terms were used: "RILI" [All fields] AND "radiotherapy" [All fields] AND "radiation pneumonia" [All fields]. This review contains a detailed introduction into the mechanism, diagnosis, and treatment of RILI, and we synthesized and analyzed these papers to form a clearly logical and normative suggestion to guide clinical application. The specific screening method is shown in Table 1.

Table 1 The search strategy summary

\begin{tabular}{ll}
\hline Items & Specification \\
\hline Date of Search (specified to date, month and year) & 1995 to 2021 \\
Databases and other sources searched & the PubMed website \\
Search terms used (including MeSH and free text search terms & "RILI" [All fields] AND "radiotherapy" [All fields] AND "radiation \\
and filters) & pneumonia" [All fields] \\
Timeframe & The development of RILI: the mechanism, diagnosis, and treatment \\
$\begin{array}{l}\text { Inclusion and exclusion criteria (study type, language } \\
\text { restrictions, etc.) }\end{array}$ & $\begin{array}{l}\text { The clinical trial, literature review, and review paper; no language } \\
\text { restriction }\end{array}$ \\
$\begin{array}{l}\text { Selection process (who conducted the selection, whether it was } \\
\text { conducted independently, how consensus was obtained, etc.) }\end{array}$ & $\begin{array}{l}\text { The first author conducted the selection independently, and obtained } \\
\text { the consensus by sending to other authors }\end{array}$ \\
Any additional considerations, if applicable & N/A \\
\hline
\end{tabular}

RILI, radiation-induced lung injury; MeSH, Medical Subject Headings. 


\section{Mechanism exploration}

After irradiation, tissues are damaged either directly or indirectly. Ionizing radiation leads to direct deoxyribonucleic acid (DNA) damage, including base deletion, DNA singlestrand break (SSB), and DNA double-strand break (DSB) (2). These damages can be detected by various technical methods involving single-cell gel electrophoresis (known as a comet assay) and pulsed-field gel electrophoresis (PFGE), etc. Tissue repair mechanisms, such as base excision repair (BER), nucleotide excision repair (NER), homologous recombination repair (HRR), and non-homologous endjoining (NHEJ), are seen to relieve tissue injury. The bulk of injuries are repaired through cellular repair functions, and residual damaged cells are induced to apoptosis or mutation, which may contribute to tumor development.

Another indirect damage of DNA is activated by reactive oxygen species (ROS), which are primarily produced by mitochondrial oxidative metabolism to participate in the growth, differentiation, progression, and death of cells in the biological process (6). Furthermore, water molecules of irradiated cells are ionized to generate an excessive number of ROS, including superoxide, hydrogen peroxide, hydroxyl radicals, and nitrogen species (NGS), which indirectly cause DNA damage $(7,8)$. Except for analogous nuclear DNA damage with direct impacts, ROS also induces mitochondrial DNA (mtDNA) damage, which leads to protein carbonylation, lipid peroxidation, increased oxidative metabolism, and enhanced rates of spontaneous gene mutations and neoplastic transformation. Moreover, DNA damage and ROS activation stimulate series of intracellular signaling pathways and signal factors including transforming growth factor- $\beta$ (TGF- $\beta$ ), platelet-derived growth factor (PDGF), and interleukin 1 (IL-1) (9).

The source of cellular ROS can be divided into two main categories: first, normal biological processes, mainly mitochondrial oxidative metabolism, release ROS as a byproduct or a waste product of various necessary reactions. In addition, there are other processes in the cellular response to xenobiotics, cytokines, and bacterial invasion, which intentionally generate ROS, either in molecular synthesis or in breakdown as part of a signal transduction pathway or a cell defense mechanism. Numerous studies found that ROS have a substantial influence on nuclear factor kappa B (NF$\kappa \mathrm{B})$ signaling pathways $(6,10)$. NF- $\kappa \mathrm{B}$, a transcription factor that participates in immune and inflammatory responses, is activated by IкB kinase (IKK) complex, which consists of the IKK $\alpha$ and IKK $\beta$ catalytic subunits and the IKK $\gamma$ regulatory subunit, catalyzing the phosphorylation of I $\kappa \mathrm{Bs}$ polyubiquitination and subsequent degradation by the $26 \mathrm{~S}$ proteasome to translocate to the nucleus, thereby binding DNA and activating gene transcription. Additionally, tumor necrosis factor receptor (TNFR), Toll-like receptor (TLR), and IL-1 are also involved in this process. The activation and nuclear translocation of NF- $\mathrm{\kappa B}$ can stimulate inflammatory-mediated effects and result in the occurrence of chemokines, cytokines [intercellular adhesion molecule 1 (ICAM-1), vascular cell adhesion molecule-1 (VCAM1 ), and endothelial-leukocyte adhesion molecule 1 (ELAM1)]. In the lung tissue, ROS generation is derived from endothelial cells, neutrophils, eosinophils, alveolar macrophages, and alveolar epithelial cells (AECs). ROS generation causes damage to the endothelial barrier, as well as an increase in vascular permeability and trans-endothelial migration of enormous leukocyte (Figure 1).

The development of RILI is divided into various stages by different scholars. Arroyo-Hernández et al. distributed RILI to five phases based on the molecular changes: early phase, latent phase, exudative phase, intermediate phase, and fibrotic phase (4). Hanania et al. posited that RILI is made up of three stages: latent phase, acute-phase pneumonitis, and late-phase pulmonary fibrosis (3). Most people regarded this classification as the classic channel, and the sporadic classification also has been raised, which includes other types of pneumonia such as hypersensitivity pneumonitis.

We divided the development of RILI into two stages. In the early stage (RP), damage-associated molecular pattern molecules (DAMPs) are released from cells to recruit many immune effector cells to accumulate the damage of lung tissue and contribute to tissue remodeling. Under the induction of intercellular cell adhesion molecule-1 (ICAM-1) and platelet endothelial cell adhesion molecule-1 (PECAM-1/CD31), neutrophils and macrophages arrive one after another and release IL-3, IL-6, IL-7, TNF- $\alpha$, and TGF- $\beta$ to produce an inflammatory reaction. In the late stage (RLF), T helper 2 (Th2) cells participate in the profibrotic process. Neutrophils and macrophages induced profibrotic effects via the secretion of TGF- $\beta$, IL-6, and PDGF. Blood monocytes are recruited to lungs and differentiate into fibroblasts and myofibroblasts. Neutrophils secrete elastase and matrix metalloproteinases to contribution to accumulation ECM. Finally, pulmonary fibrosis occurs. 


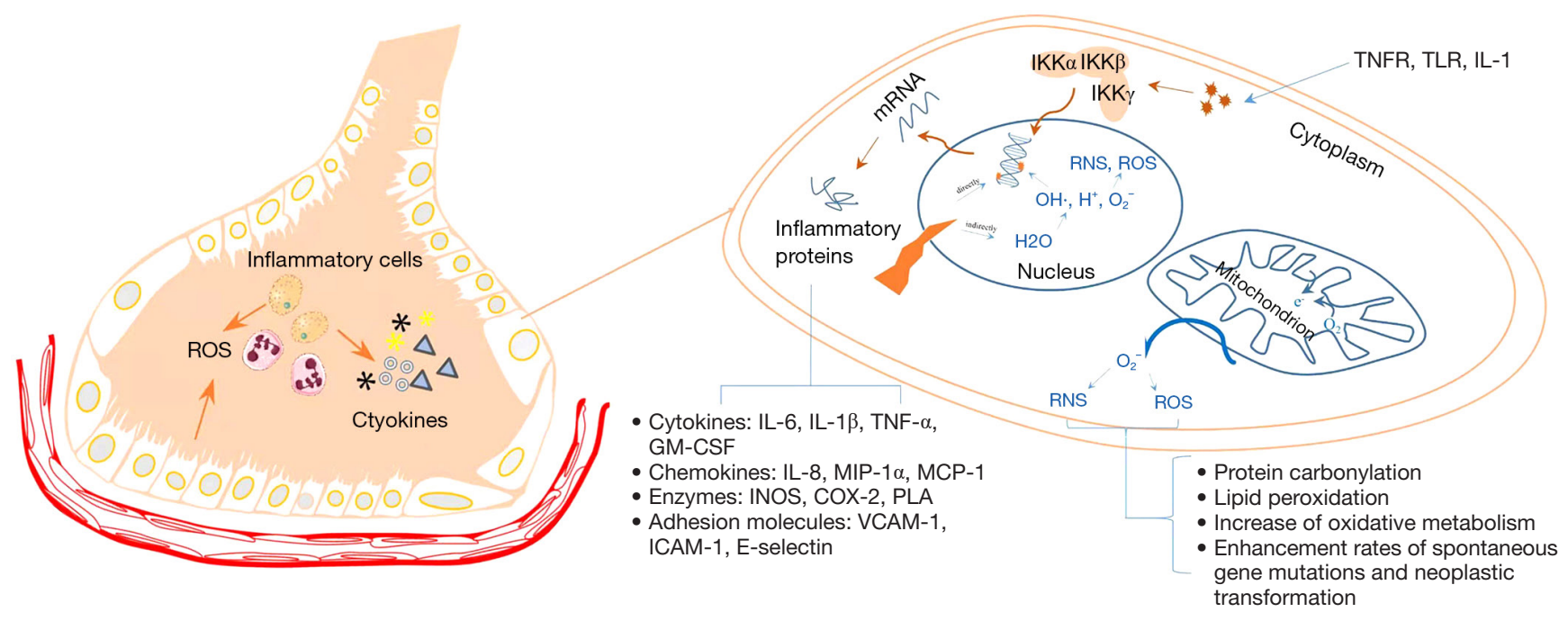

Figure 1 The mechanism of DNA damage and ROS generation. ROS, reactive oxygen species; RNS, reactive nitrogen species; DNA, deoxyribonucleic acid; mRNA, messenger RNA; IKK, IкB kinase; TNFR, tumor necrosis factor receptor; TLR, Toll-like receptor; IL, interleukin; TNF- $\alpha$, tumor necrosis factor- $\alpha$; GM-CSF, granulocyte-macrophage colony stimulating factor; MIP-1 $\alpha$, macrophage inflammatory protein-1 $\alpha$; MCP-1, monocyte chemoattractant protein-1; INOS, inducible nitric oxide synthase; COX-2, cyclooxygenase-2; PLA, polylactic acid; VACM-1, vascular cell adhesion molecule-1; ICAM-1, intercellular adhesion molecule 1.

\section{Cellular changes}

\section{Epithelial and endothelial cells}

The human alveolar epithelium consists of type-I and II pneumocytes, which make up $90 \%$ and $10 \%$ of cells in the alveoli, respectively. Ionizing radiation induces direct DNA damage as well as the generation of reactive oxygen, which contribute to the apoptosis of the sensitive type-I pneumocytes within minutes (11). As the precursors of type-I cells, type-II pneumocytes increase and drive reepithelialization of the alveolus. Moreover, ionization of water molecules generates ROS. ROS also causes edema of the alveolar walls, increased vascular permeability, and exudation of proteins into the alveolar space, which further reduces the alveolar septa. The process of epithelial and endothelial cell damage manifests as damage to the connections between cells, accompanied by impaired regulation of myofibroblasts and deposition of excessive ECM (12). A large number of blood exudate and inflammatory cells accumulate in the alveolar cavity, which leads to intra-alveolar edema and aggregates numerous fibroblasts and induces their differentiation into myofibroblasts. Activated myofibroblasts then secrete angiotensin and hydrogen peroxide, which in turn induce AECs apoptosis (13). The following cytokines are released during this process: tumor necrosis factor- $\alpha$ (TNF- $\alpha$ ), IL-1, IL-6, high-molecular-weight mucin-like antigen KL-6,
PDGF- $\beta$, and basic fibroblastic growth factor (bFGF) $(14,15)$.

\section{Th1 and Th1 cells}

The involvement of lymphocytes plays an important role in radiation-induced early lung inflammation, which has been demonstrated by accumulating evidence (16-18). Th1 cells secrete IFN- $\gamma$, which contributes to significant antifibrosis and immunomodulatory activity. Th2 cells mainly secrete IL-4 and IL-13, which co-stimulate with TGF- $\beta 1$ in collagen synthesis (19). An imbalance between Th1 and Th2 cells promotes the development of RP. T regulatory (Treg) cells can express a series of inflammatory chemokines receptors (e.g., CCR2, CCR4, and CCR5) (20), which strengthen the inflammatory response after tissue injury. Furthermore, Treg cells also participate in the promotion of fibrocyte accumulation, promotion of epithelialmesenchymal transition (EMT), modulation of Th1/Th2 balance, and suppression of Th17 responses. Immune-cellmediated immune response can drive the development of RP, suggesting the application of immune checkpoint inhibitors (ICIs) for tumor treatment (21-23).

\section{Macrophages and fibroblasts}

Macrophages and fibroblasts are activated under the inducements of ILs, TNF, TGF, and PDGF to synthetize 
ECM. Macrophages are divided into two types, including classic-activated macrophages (M1), which is regulated by Th1-derived cytokine IFN- $\gamma$, and bypass-activated macrophages (M2), which is regulated by Th2-derived cytokines IL-4 and IL-13. Macrophages have been implicated in carcinogenesis, angiogenic switch, local invasion, and metastasis (24). In the early stage of RILI, M1 macrophages secrete pro-inflammatory cytokines to induce inflammation and produce massive ROS (via a ROS-induced cascade) to further impair lung tissue. In the late stage of RILI, M2 macrophages secrete profibrotic cytokines to promote the development of RLF (25). M2 macrophages express Arg1 , which controls L-proline production that is required for collagen synthesis by activated myofibroblasts to induce mesenchymal transition of epithelial cells. The TGF- $\beta$ secretion of M2 macrophages has a similar effect (26). These cytokines promote radiation-induced fibrosis in the late stage.

Under the action of chemokines, various types of inflammatory cells, such as lymphocytes, macrophages, neutrophils, and mononuclear cells, proliferate, aggregate, and migrate into the pulmonary interstitial tissue, release various cytokines, and cause alveolar edema, eventually lead to the occurrence of pulmonary fibrosis. In addition, under the inducing function of cytokines including TGF- $\beta 1$, PDGF, and stromal cell derived factor-1 (SDF-1/CXCL12), fibroblasts and myofibroblasts accumulate in the three ways: in situ proliferation, epithelial-mesenchymal transformation, and derivation from bone marrow to synthetize and secrete collagen, which is the main component of the ECM (27). After radiation, lung tissue produces massive fibroblasts and myofibroblasts, causing extensive production of collagen, infiltration of inflammatory cells, and remodeling of the ECM (28). Fibrosis of the alveolar septum subsequently leads to extensive occlusion of the alveoli.

\section{The changes of cytokines}

\section{TGF- $\beta$}

As a powerful pro-fibrotic growth factor, TGF- $\beta$ has some functions, including inducing proliferation and differentiation of fibroblasts, promoting synthesis of collagen by fibroblasts, inhibiting synthesis of collagenase and plasminogen activator, and aggregating considerable amounts of inflammatory cells and cytokines (PDGF, TNF- $\alpha$, IL-4, etc.). Increased TGF- $\beta$ levels after radiotherapy accompanies elevated collagen IV gene expression (28), which leads to septal thickening and is indicative of microvascular injury. By stimulating metalloproteinase inhibitors (TIMPs), TGF- $\beta$ inhibits collagen catabolism, which results in collagen accumulation and conversion of fibroblasts into myofibroblasts, leading to lung architecture remodeling. Increased fibroblasts with the loss of functional cells lead to loss of respiratory capacity, tissue atrophy, and necrosis. When TGF- $\beta$ is overexpressed in tumor tissues, it will facilitate the process of EMT (29).

\section{IL-4 and IL-13}

IL-4 is mainly expressed by Th2 cells, and also secreted by innate immune cells including mast cells, basophils, and eosinophils via the signal transducer and activator of transcription 5/GATA binding protein 2 (Stat5/GATA2) or GATA1 pathway, to drive the production of macrophages, fibroblasts, and epithelial cells $(12,30)$. IL-4 participates in the differentiation of Th2 cells and inhibits the activities of Th1 cells. IL-4 stimulates arginase activity and converts arginine to ornithine in macrophages. Ornithine serves as a precursor of polyamines and collagen, and ultimately contributes to the deposition of ECM (31). IL-13 is mainly secreted by Th2 cells, and has similar functions as IL-4. IL-4 and IL-13, as important pro-fibrotic cytokines, are required for the initiation and maintenance of pulmonary fibrosis, as well as other organs (liver, skin) (32,33).

\section{IFN- $\gamma$}

IFN- $\gamma$ is produced by Th1 cells and plays a key role in antifibrosis and immunomodulatory effects. IFN- $\gamma$ can induce M1 macrophages to express a high level of inflammatory cytokines, such as IL-2, IL-23, and nitric oxide (NO), and promote inflammatory processes (30). In addition, related research has reported that IFN- $\gamma$ has anti-fibrotic effects. IFN- $\gamma$ treatment inhibits the TGF- $\beta$-induced phosphorylation of Smad3 and its attendant events, and mediates the downregulation of bleomycin (BL)-induced overexpression of TGF- $\beta$, which is a powerful pro-fibrotic growth factor in BL-induced lung fibrosis mouse model $(34,35)$, it suggests that IFN- $\gamma$ has a favorable influence on the anti-fibrotic progress of RP.

\section{Prostaglandin E2 (PGE2)}

PGE2 is a pro-inflammatory mediator in a variety of diseases. It can inhibit the secretion of TGF- $\beta$ and the differentiation of fibroblasts into myofibroblast in lung tissue to effect anti-fibrotic activity (36). By stimulating E prostanoid 2 (EP2) and EP4 receptors and downstream protein kinase $(\mathrm{PK})$ or exchange protein, cyclic adenosine monophosphate (cAMP) is activated to mediate anti-fibrotic 
actions of PGE2 (37). PGE2 are classified as an endogenous negative regulator of forkhead box M1 (FOXM1), which drives the activation of lung fibroblasts to inhibit radiation-induced fibrosis, including decreasing fibroblast chemotaxis, fibroblast proliferation, fibroblast growth factor receptor expression, collagen synthesis and myofibroblast differentiation, and increasing collagen degradation (38-40).

In conclusion, the pathological change of RILI is mediated by a series of cells and cytokines. Radiation induces DNA damage, and the generation of reactive oxygen stimulates the activation of inflammatory pathways. Direct damage to epithelial alveolar and endothelial cells leads to pro-inflammatory and pro-fibroblast activity. The release of a variety of cytokines drives the action of macrophages and the differentiation of fibroblasts. Th 1 and Th2 cells participate in the polarization and infiltration of cytokines. TGF- $\beta$ plays a key role in this process, and IFN- $\gamma$, macrophages, and PGE2 have a positive impact in inhibiting lung fibrosis. However, IL-4 and IL-13 secreted by Th2 cells exert a contrary effect. Fibroblasts proliferate and differentiate to drive collagen synthesis and ECM deposition under the influence of TGF- $\beta$, IL-4, IL-13, IFN- $\gamma$, PGE2, and others. A variety of chemokines promote the development of RILI.

Certainly, there are many microvariations that have not been found or demonstrated; for example, more signaling pathways are yet to be explored. Building related animal models can assist in the investigation of further mechanisms, which can be utilized to conduct clinical practice.

\section{The diagnosis of RILI}

RILI is divided into two stages: RP in the early stage and RLF in the late stage. RP most frequently occurs within 1-6 months, while the onset of RLF occurs as a late toxicity beyond 6 months. There are non-specific symptoms that occur in both stages: fever, chest pain, cough, fatigue, tachypnea, and others. Patients may express aggravation of original respiratory symptoms and/or new clinical manifestations. For example, the frequency and degree of cough and phlegm increases with severe symptoms, such as hypoxemia and newly occurred respiratory failure $(4,41)$. Physical examination may include pleural friction rub or moist rales. Laboratory testing sometimes reveals a high percentage of neutrophils. Most relevant, imaging classically demonstrates findings most typically consist of development of a diffuse a ground-glass opacity or masslike consolidation in the field of radiation treatment, with bronchial pull and scar-like changes (42). RLF has similar clinical symptoms and physical examination, but fever is rare. Imaging cross-sectional imaging classically shows ventilated bronchial signs, strip shadows, lung consolidation shadows, or honeycomb changes within the irradiated lung tissue.

According to the clinical manifestation and imaging performance, some researchers have divided RILI into four grades to guide treatment. There are many classifications in the field; currently, the most commonly used criteria are the Common Terminology Criteria for Adverse Events (CTCAE-5.0) (Table 2). Here, RILI is graded based on the existence and severity of the clinical symptoms, whether pneumonitis intervention is required, and on the extent of pulmonary fibrosis and accompanying symptoms. RILI could be diagnosed based on the clinical manifestations and imaging. RP usually occurs in the radiotherapy area and rarely accumulates in distant lung tissues. Only patients with a history of radiotherapy exposure may develop RP. $\mathrm{RP}$ is often accompanied by bacterial, viral, or fungal infections, such as Pneumocystis carinii, and is treated with antibiotics together. Immune pneumonitis should be distinguished from RP, and it also manifests as interstitial lung changes, but appearance of it is irregular in time, and is not limited to one place, often accompanied by immune diseases of other organs or tissues. Malignant lung tumor patients may experience specific pneumonitis when immunotherapy immunomodulatory agents [PD-1, PD-L1, and cytotoxic T-lymphocyte-associated antigen 4 (CTLA-4) inhibitors] are used in the concurrent or adjuvant setting to treat disease. Immune-related pneumonitis also presents clinically with cough and/or shortness of breath, as well as lung interstitial findings on cross-sectional imaging. Further study is required to help clinicians better distinguish between RILI and other pulmonary ailments to help guide clinical decisions making surrounding management options, such as discontinuation of immunotherapies, use of steroids or prompt initiation of antibiotics (43) In addition, RP should also be differentiated from other lung diseases such as tumor progression, pulmonary embolism, and tuberculosis.

\section{The treatment of RILI}

RILI is treated according to the grade conditions: grade 1 RILI is usually not treated but actively monitored in case symptoms in case developing develop; and $\geq$ grade 2 patients who experience classical clinical manifestation and imaging 
Table 2 The RILI grade in CTCAE-5.0

\begin{tabular}{|c|c|c|c|c|c|}
\hline Adverse event & Grade 1 & Grade 2 & Grade 3 & Grade 4 & Grade 5 \\
\hline $\begin{array}{l}\text { Pulmonary fibrosis } \\
\text { (definition: a disorder } \\
\text { characterized by the } \\
\text { replacement of the lung } \\
\text { tissue by connective tissue, } \\
\text { leading to progressive } \\
\text { dyspnea, respiratory } \\
\text { failure, or right heart failure) }\end{array}$ & $\begin{array}{l}\text { Radiologic } \\
\text { pulmonary } \\
\text { fibrosis }<25 \% \\
\text { of lung volume } \\
\text { associated } \\
\text { hypoxia }\end{array}$ & $\begin{array}{l}\text { Evidence of } \\
\text { pulmonary } \\
\text { hypertension; } \\
\text { radiographic } \\
\text { pulmonary } \\
\text { fibrosis } 25-50 \% \\
\text { associated with } \\
\text { hypoxia }\end{array}$ & $\begin{array}{l}\text { Severe hypoxia; } \\
\text { evidence of } \\
\text { right-sided } \\
\text { heart failure; } \\
\text { radiographic } \\
\text { pulmonary } \\
\text { fibrosis }>50-75 \%\end{array}$ & $\begin{array}{l}\text { Life-threatening } \\
\text { consequences (e.g., } \\
\text { hemodynamic/pulmonary } \\
\text { complication); intubation } \\
\text { with ventilatory support } \\
\text { indicated; radiographic } \\
\text { pulmonary fibrosis }>75 \% \\
\text { with severe honeycombing }\end{array}$ & Death \\
\hline
\end{tabular}

RILI, radiation-induced lung injury; $A D L$, activities of daily living.

Table 3 RILI treatment

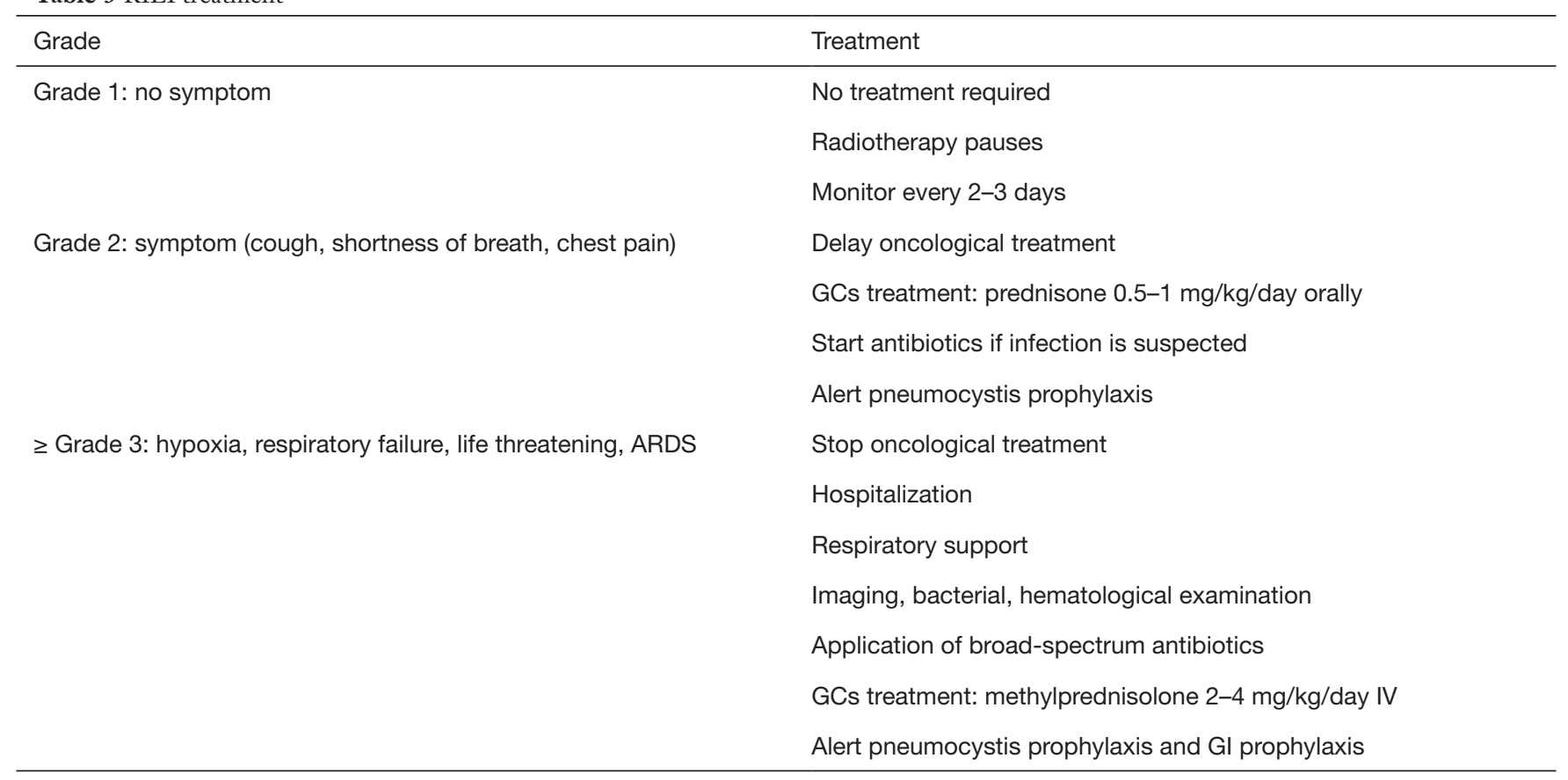

RILI, radiation-induced lung injury; GCs, glucocorticoids; ARDS, acute respiratory distress syndrome; GI, gastrointestinal; IV, intravenous.

changes prompting the need for timely treatment. The first step is to cease anti-tumor therapy, followed by comprehensive measures, including anti-inflammatory (e.g., oral prednisone $0.5-1 \mathrm{mg} / \mathrm{kg} /$ day) and other symptomaticbased treatments. Drugs should also be used to prevent. In the case of prolonged steroid use, pneumocystis prophylaxis. and gastrointestinal (GI) stress-ulcer prophylaxis should be considered. Patients of $\geq$ grade 3 should be evaluated for urgent hospitalization and supplemental oxygen requirements (Table 3). 
Table 4 Glucocorticoid action efficiency reference table

\begin{tabular}{|c|c|c|c|c|c|}
\hline Category & Drug & $\begin{array}{l}\text { Anti-inflammatory } \\
\text { equivalent dose (mg) }\end{array}$ & $\begin{array}{l}\text { Anti-inflammatory } \\
\text { strength }\end{array}$ & $\begin{array}{l}\text { Serum half-life } \\
\qquad(\min )\end{array}$ & $\begin{array}{c}\text { Pharmacological } \\
\text { half-life }(\mathrm{h})\end{array}$ \\
\hline \multirow[t]{2}{*}{ Short efficiency } & Cortisone & 25 & 0.8 & 30 & $8-12$ \\
\hline & Hydrocortisone & 20 & 1 & 90 & $8-12$ \\
\hline \multirow[t]{2}{*}{ Medium efficiency } & Prednisone & 5 & 4 & 60 & $12-36$ \\
\hline & Methylprednisolone & 4 & 5 & 180 & $12-36$ \\
\hline \multirow[t]{2}{*}{ Large efficiency } & Dexamethasone & 0.75 & 25 & $100-300$ & $36-54$ \\
\hline & Betamethasone & 0.75 & 25 & $100-300$ & $36-54$ \\
\hline
\end{tabular}

Suitable doses and treatment with GCs drugs are often used to heal RILI patients, which has been confirmed by multiple studies $(4,44,45)$. GCs exert inhibitory effects on a series of pro-inflammatory genes including cytokines, chemokines, and receptors (46). NF- $\kappa \mathrm{B}$ and activator protein-1 (AP-1) are inhibited to reduce histone acetylation, chromatin remodeling, and RNA polymerase II actions. GCs can inhibit mitogen-activated protein kinase (MAPK) signaling pathways via the induction of mitogen-activated protein kinase (MAPK) phosphatase-1 (MKP-1), which has also important anti-inflammatory activity (47). Some studies have shown that the binding of GCs receptor (GR) homodimers and GC response elements can promote GC gene expression enhancement, which is known as transactivation (TA) $(48,49)$. Also, monomeric GR binds transcription factor (e.g., NF- $\mathrm{kB}$ or AP-1) to mediate GR gene regulation.

There are various GC drugs with different efficiencies and mechanisms (some examples are shown in Table 4). Patients with $\geq$ grade 2 are suggested to take GC drugs. Mild symptomatic patients are suggested to take prednisone $0.5-1 \mathrm{mg} / \mathrm{kg} /$ day orally, while severe symptomatic patients are suggested to take methylprednisolone $2-4 \mathrm{mg} / \mathrm{kg} / \mathrm{day}$ by intravenous injection over 6 weeks. When symptoms and imaging changes release and stabilize, patients can begin to gradually reduce drug treatment to avoid rebounding (4). RILI therapy should be early, sufficient, and based on individual principles to get achieve treatment results. As powerful anti-inflammatory and immunosuppressive drugs, long-term and high-doses use of GCs will lead to a high incidence of adverse effects. Also, dosage, dosing regime, specific drug used, and individual patient variability are also important influencing factors (47). Inappropriate application of GCs could cause various systemic disorders, including cardiovascular system, central nervous system (CNS), eye, immune system, skin, and others. Common side effects of GCs are glaucoma, cataracts, tissue atrophy and extended wound healing, adrenal suppression, and osteoporosis (50). During the application of GCs antibiotics should be used if infection is suspected. Other adjutant therapies including oxygen inhalation, atomization, and nutritional support should be applied.

Other than GC drugs, other new drugs used to treat RILI have also been reported. Some reports have noted that mesenchymal stem cells (MSCs) can prevent lung fibroblasts from myofibroblasts to inhibit lung fibrosis. Human umbilical cord MSCs (HUMSCs) co-cultured with irradiated human lung fibroblasts (HLFs) could attenuate $\mathrm{Wnt} / \beta$-catenin signaling in HLFs, which is activated by radiation and inhibits frequently rearranged in advanced T cell lymphomas-1 (FRAT1) expression, as a protein of increasing $W n t / \beta$-catenin signaling, to retard fibrosis process (51). TGF- $\beta 1$ can regulate the transformation of fibroblasts into myofibroblasts and induce type II AECs to develop EMT in myofibroblasts via the phosphorylation of the Smad2/3 signaling pathway. Hepatocyte growth factor (HGF) can attenuate BL-induced EMT in type II AECs with increasing expression of Smad7 upon binding to c-Met, and upregulate the expression of matrix metalloproteinases to promote the apoptosis of myofibroblasts. PGE2 can inhibit the synthesis of collagens by elevating intracellular cAMP levels, and inhibit activation and proliferation of fibroblasts (40). It has been reported that irradiated rats injected into human adipose tissue-driven MSCs increase 
secretion of HGF and PGE2 and decrease the expression of TNF- $\alpha$ and TGF- $\beta 1$. MSCs were also suggested to potentially exert lung fibrosis-limiting activity (52).

Pentoxifylline (Ptx) is an ethyl xanthine derivative that has been demonstrated to exert a prostacyclin-like effect by inhibiting platelet aggregation and enhancing microvascular blood flow. Some studies have also shown that Ptx can promote immunomodulatory and anti-inflammatory activations by inhibiting TNF and IL-1 $(53,54)$. Moreover, Ptx has been suggested to have a potential function in alleviating damage of RILI. Ozturk et al. conducted a randomized clinical study and found that patients with Ptx in the irradiation groups have a marked decrease in severe RILI $(\mathrm{P}<0.05)(55)$. Moreover, they suggested a concomitant use dose of Ptx with radiation of $1,200 \mathrm{mg} /$ day, in order to achieve minimal side effects. Other studies have demonstrated the Ptx treatment effect in RILI (56,57); a positive outcome was observed in a randomized, placecontrolled clinical trial that Ptx combined with alphatocopherol [vitamin E (Vit E)] in the treatment of lung fibrosis after radiation.

Azithromycin, as a macrolide antibiotic, exhibits immunomodulatory and anti-inflammatory effects. Azithromycin inhibits inflammatory signaling by suppressing the expression of lipopolysaccharide (LPS)-induced macrophage-driven chemokines (MDC) via the c-Jun N-terminal kinase (JNK) and NF- $\mathrm{KB}$ p65 pathway, and suppressing LPS-induced IFN-inducible protein-10 (IP-10/CXCL10) to achieve asthmatic airway inflammation and hypersensitivity via the MAPK-JNK/ERK and NF- $\kappa B$ p65 pathways (58). Azithromycin also has a different influence on cell levels including promoting macrophage polarization, inhibiting the effects of neutrophils, and inhibiting autophagosome clearance. Moreover, it can downregulate the expression of IL-1 $\beta$, IL- 6 , TNF- $\alpha$, TGF- $\beta 1$, and $\alpha$-SMA, which all are pro-fibroblasts and pro-inflammatory cytokines (59). However, we have not chosen suitable doses to guide treatment of RILI.

Numerous studies have shown that multiple drugs play a positive role in RILI, but they rarely apply to clinical practice. Angiotensin-converting enzyme inhibitors (ACEIs) can release pulmonary collagen deposition and fibrosis in the process of radiation (60-62). Berberine applied to non-small cell lung cancer (NSCLC) patients can reduce the prevalence of acute RILI, which may decrease the expression of ICAM- 1 and TGF- $\beta 1$ (63). The application of anti-TGF- $\beta$ also prevents RILI by inhibiting the TGF- $\beta$ receptor.
Amifostine, as a radioprotective agent, reduces irradiation-induced DNA damage by eliminating superoxide dismutase 2 (SOD2) and lowers the oxygen concentration around normal tissue (64). The above-mentioned drugs may have positive influences on the mechanism of RILI; however, these effects have been rarely seen in clinical practice. More research is needed to estimate their actual value.

\section{Predisposing factor of RILI}

\section{Treatment factors}

Radiation therapy can cause damage to normal tissues around tumors cells. Currently, a variety of radiotechnologies [e.g., intensity-modulated radiation therapy (IMRT), volumetric arc radiotherapy (VMAT), and imageguided radiotherapy (IGRT)] applied in clinical practice have helped improve upon this problem, but the incidence of RILI has been reported in up to $30 \%$ of patients. Many factors can impact the outcome, including radiation dose, fractionation, lung dose, and combined treatment. Based on fractionation, fraction dose, and the effects of early- and late-response tissues, radiotherapy methods can be divided into conventional fractionated radiotherapy (2 Gy/Fra/day, five times/week), hyperfractionated radiotherapy (1.2 Gy $\times 2 /$ day), and hypofractionated radiotherapy (3-10 Gy/Fra).

Conventional fractionated therapy is the primary method for locally advanced patients. Accelerated hyperfractionated radiotherapy can be applied for late-stage patients to obtain a good local control rate by escalating total dose and shortening the overall time (65). Stereotactic body radiation therapy (SBRT), as a hypofractioned way of highdose radiation delivered over 1-5 treatments, has prominent advantages for medically inoperable early-stage lung cancer patients (66). A related analysis of two randomized trials indicated that compared to surgery, SBRT for I stage NSCLC showed analogous local control and overall survival rates.

The percentage of lung receiving a dose in excess of 20 Gy (V20) and the mean lung dose (MLD) have been viewed as predictors of RILI after irradiation, and numerous trials have been performed to confirm this. Patients who received higher V20 values showed high grades radiation pneumonitis $(\mathrm{P}<0.001)$. V20 has also been shown to be a predictor for $\geq$ grade 2 RP (67). Another retrospective analysis for locally advanced NSCLC treated with concurrent chemoradiotherapy (CCRT) reported that V20 $\geq 26 \%$ was 


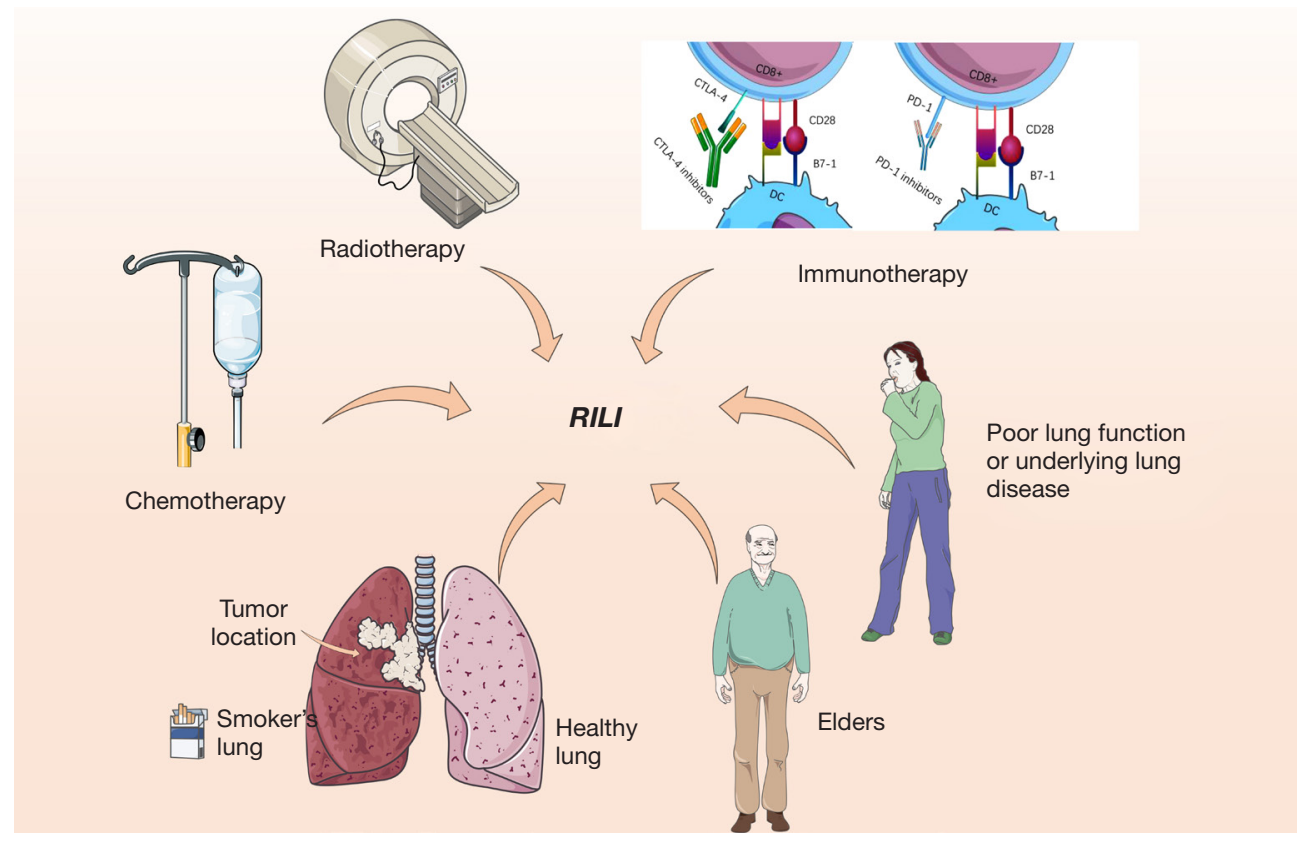

Figure 2 The predisposing factor of RILI. CTLA-4, cytotoxic T-lymphocyte-associated antigen 4; DC, dendritic cell; RILI, radiationinduced lung injury.

an independent predictive factor for $\geq$ grade $3 \mathrm{RP}$, and suggested that the V20 dose constraint should be less than $30-35 \%$ for CCRT patients (68). Some researchers have used the large multi-institutional analysis standard of Kwa to analyze a logistic regression fitted to RP $v s$. MLD date from all published large-sized studies. They found that an absolute safe MLD for which there is no risk for RILI is non-existent, and the risk-benefit ratio in individual cases should be used to drive clinical decision making (69). In addition to MLD and V20, V5 and V30 have also been correlated with RILI (Figure 2).

Some reports have indicated that radiation associated with other treatment methods may impact the incidence of RILI. Radiotherapy connected with concurrent or sequential chemotherapy is often used to treat locally advanced lung cancer. However, this multimodality approach is associated with more serious adverse responses than radiotherapy alone. Fournel et al. developed a phase III clinical trial regarding concurrent or sequential chemoradiotherapy (cisplatin and vinorelbine) for locally advanced NSCLC patients (70). Compared with the sequential group, the concurrent group showed a better survival rate at 2, 3, and 4 years, despite lacking statistical significance. The concurrent chemotherapy arm, however, demonstrated a higher incidence of adverse events compared with the sequential group.

On the contrary, the meta-analysis of Vogelius et al. regarding the clinical risk factors for RP surprisingly reported that more adverse events occurred with sequential chemotherapy than concurrent chemotherapy (71). They speculated that the reason for this is due to the fact that the biological effects of radiation continue for several weeks after the last fraction, as well as the lower dose chemotherapy in sequential groups, and suggested that more data is needed to explore interaction between chemoradiotherapy.

Radiotherapy and immunotherapy have become a focal point in recent years. However, the interaction between both therapies in terms of the mechanism, application, and toxicity remains unclear. A study has observed that radiotherapy activates innate and adaptive immune responses by exposure to immunogenic molecules, thus releasing damaged-associated molecular patterns (DAMPs) including uric acid, S100 protein, adenosine triphosphate (ATP), high mobility group protein B1 (HMGB1), or tumor antigens (72).

ICIs are increasingly used as effective cancer treatments with intensifying immune recognition and killing of tumor cells (73). The application of radiation and immunotherapy has clearly improved the total survival rate, which has been 
confirmed in multiple large trials (74). In the KEYNOTE-001 trial, 24 of 97 patients who received radiotherapy had a median of 11.5 months prior to ICI. Researchers observed pulmonary toxicity in $63 \%$ of combined therapy patients versus $40 \%$ in patients who did not receive radiotherapy. The PACIFIC trial, which included 713 patients who received sequential ICI treatment between 1-42 days after chemoradiotherapy, also observed that pneumonitis was more frequent in the combined treatment than in radiotherapy alone (33.9\% vs. $24.8 \%$ ). Thus, with the wider application of immunotherapy in clinical practice, we should be more alert to the occurrence of highgrade RILI $(75,76)$.

Currently, targeted drugs have been used as effective cancer treatments, mainly including vascular endothelial growth factor receptor (VEGFR) pathway (bevacizumab), and epidermal growth factor receptor (EGFR) pathway (necitumumab). VEGF can induce the growth and survival of the vascular endothelium and promote angiogenic growth, modulate the immune microenvironment, and inhibit the differentiation and function of diverse immune cells, such as dendritic cells, macrophages, and others (77). EGFR can promote proliferation, invasion, metastasis, anti-apoptotic signaling, and angiogenesis in tumor cells. A meta-analysis regarding EGFR tyrosine kinase inhibitors (EGFR-TKIs) treatment for late-stage lung cancer observed an incidence of $\geq$ grade 3 pneumonitis of up to $0.9 \%$ [95\% confidence interval (CI): $1.0-2.4 \%]$, with a mortality of $13.0 \%$ (95\% CI: 7.6-21.6\%). They concluded that treatment with EGFR-TKIs was associated with a significantly increased risk of pneumonitis (78).

We did not find surgery to be associated with a risk of RILI. However, the type of surgery may impact the outcome. A recent study found overall survival was improved in patients receiving tri-modality therapy with chemoradiotherapy, followed by lobectomy, compared with chemoradiotherapy alone (79). The overall survival was not improved for patients undergoing pneumonectomy. The surgery group showed lower pulmonary toxicity than the chemoradiotherapy group (9\% vs. 14\%). We hope there are more studies exploring this with stronger results.

\section{Patient factors}

Age, comorbidities, tumor location, and smoking habits are all RP risk factors. Among these, smoking habit is surprisingly a protective factor. Apparently, radiation pneumonitis could occur more easily in older patients compared with younger patients because of weak pulmonary function and more complications. However, a retrospective study by Moreno et al. of late-stage NSCLC patients indicated that patients with chronic obstructive pulmonary disease (COPD) experienced higher pulmonary toxicity than patients without complications (80). They believed that COPD was independently associated with $\geq$ grade 3 [odds ratio $(\mathrm{OR})=10.5, \mathrm{P}=0.007$ ]. Other studies have also reported similar conclusions $(81,82)$. This suggests that patients with poor lung function or underlying lung disease may be at higher risk for RILI. Some studies have investigated the interaction between tumor location in the middle or inferior part of the lung and RILI. They found that middle or inferior lung tumors had an increased risk of RILI compared with upper lung tumors $(\mathrm{P}=0.002)$ $(80,83,84)$. However, the mechanism at play here remains unelucidated. Some people believe that the difference is possibly driven by physiological motion, which enables the middle or inferior lung to accept a higher irradiation dose. A number of have studies testified that ongoing smoking could prevent RILI, and a history of smoking could be a protective factor (85-87). The reason for this may be that the lung tissue of long-term smoker is relatively insensitive to radiation or long-term cough symptoms, thus masking the clinical symptoms of RILI.

\section{Conclusions}

In this review, we investigated the mechanism of RILI occurrence, treatment methods, and predisposing factors. RILI is the result of inflammatory cells and chemokines, which has been affirmed in numerous studies. Stratifying by CTCAE 5.0 RP grade, we described various management approaches to different severities of RP and RLF. Notably, there are side effects in this process, especially for patients with long-term medication use. Newer treatments have shown promise, but application in clinical practice is still pending further study. For now, the continued study of clinical and dosimetric factors associated with RP and RLF are vital to the prevention and early diagnosis of RILI. The combination of radiotherapy and immunotherapy appears to be associated with higher rates of RP, but future research is warranted. Lastly, continued studies utilizing cell lines, animal models, and clinical trials should continue to be carried out to explore effective and suitable therapeutic 


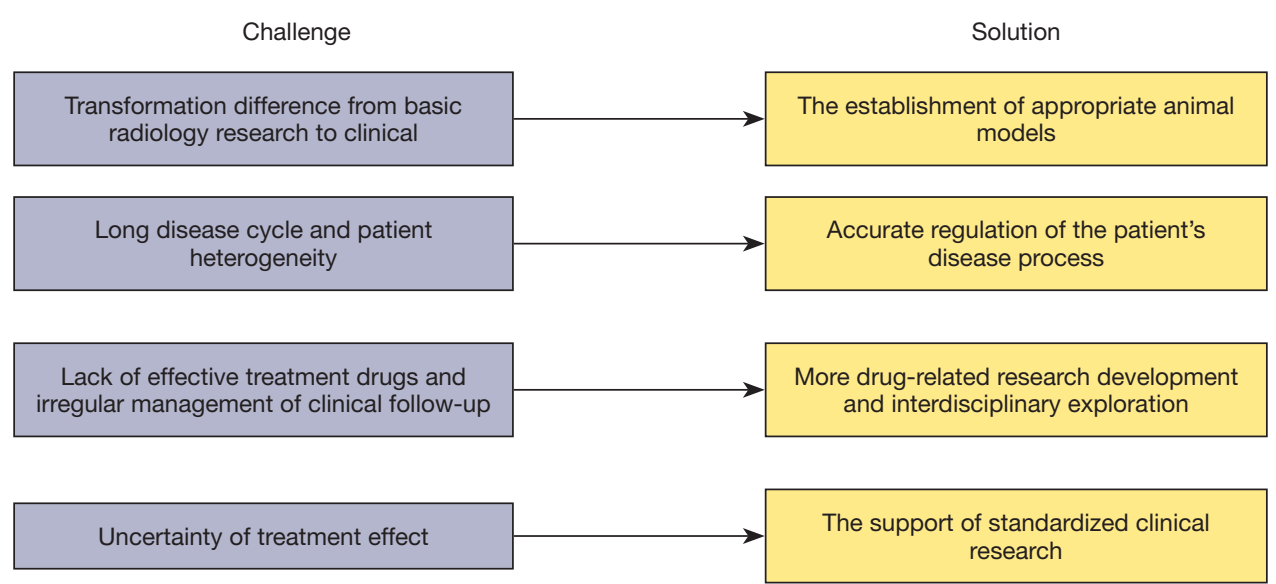

Figure 3 Challenges and solutions.

measures (Figure 3).

\section{Acknowledgments}

The authors appreciate the academic support from the AME Radiation Oncology Collaborative Group.

Funding: The study was supported by the Three Years Action to Accelerate the Development of Traditional Chinese Medicine Plan [ZY(2018-2020)-FWTX-3004].

\section{Footnote}

Reporting Checklist: The authors have completed the Narrative Review reporting checklist. Available at https:// tlcr.amegroups.com/article/view/10.21037/tlcr-22-108/rc

Conflicts of Interest: All authors have completed the ICMJE uniform disclosure form (available at https://tlcr.amegroups. com/article/view/10.21037/tlcr-22-108/coif). The authors have no conflicts of interest to declare.

Ethical Statement: The authors are accountable for all aspects of the work in ensuring that questions related to the accuracy or integrity of any part of the work are appropriately investigated and resolved.

Open Access Statement: This is an Open Access article distributed in accordance with the Creative Commons Attribution-NonCommercial-NoDerivs 4.0 International License (CC BY-NC-ND 4.0), which permits the noncommercial replication and distribution of the article with the strict proviso that no changes or edits are made and the original work is properly cited (including links to both the formal publication through the relevant DOI and the license). See: https://creativecommons.org/ licenses/by-nc-nd/4.0/.

\section{References}

1. Keffer S, Guy CL, Weiss E. Fatal Radiation Pneumonitis: Literature Review and Case Series. Adv Radiat Oncol 2019;5:238-49.

2. Giuranno L, Ient J, De Ruysscher D, et al. RadiationInduced Lung Injury (RILI). Front Oncol 2019;9:877.

3. Hanania AN, Mainwaring W, Ghebre YT, et al. RadiationInduced Lung Injury: Assessment and Management. Chest 2019;156:150-62.

4. Arroyo-Hernández M, Maldonado F, Lozano-Ruiz F, et al. Radiation-induced lung injury: current evidence. BMC Pulm Med 2021;21:9.

5. Gray JE, Villegas A, Daniel D, et al. Three-Year Overall Survival with Durvalumab after Chemoradiotherapy in Stage III NSCLC-Update from PACIFIC. J Thorac Oncol 2020;15:288-93.

6. Zhang J, Wang X, Vikash V, et al. ROS and ROSMediated Cellular Signaling. Oxid Med Cell Longev 2016;2016:4350965.

7. Terasaki Y, Ohsawa I, Terasaki M, et al. Hydrogen therapy attenuates irradiation-induced lung damage by reducing oxidative stress. Am J Physiol Lung Cell Mol Physiol 2011;301:L415-26.

8. Zhao W, Robbins ME. Inflammation and chronic oxidative stress in radiation-induced late normal tissue injury: therapeutic implications. Curr Med Chem 
2009;16:130-43.

9. Jack CI, Cottier B, Jackson MJ, et al. Indicators of free radical activity in patients developing radiation pneumonitis. Int J Radiat Oncol Biol Phys 1996;34:149-54.

10. Bonizzi G, Karin M. The two NF-kappaB activation pathways and their role in innate and adaptive immunity. Trends Immunol 2004;25:280-8.

11. Azzam EI, Jay-Gerin JP, Pain D. Ionizing radiationinduced metabolic oxidative stress and prolonged cell injury. Cancer Lett 2012;327:48-60.

12. Huang Y, Zhang W, Yu F, et al. The Cellular and Molecular Mechanism of Radiation-Induced Lung Injury. Med Sci Monit 2017;23:3446-50.

13. Lederer DJ, Martinez FJ. Idiopathic Pulmonary Fibrosis. N Engl J Med 2018;378:1811-23.

14. Kouloulias V, Zygogianni A, Efstathopoulos E, et al. Suggestion for a new grading scale for radiation induced pneumonitis based on radiological findings of computerized tomography: correlation with clinical and radiotherapeutic parameters in lung cancer patients. Asian Pac J Cancer Prev 2013;14:2717-22.

15. Selman M, Pardo A. Role of epithelial cells in idiopathic pulmonary fibrosis: from innocent targets to serial killers. Proc Am Thorac Soc 2006;3:364-72.

16. Wirsdörfer F, Cappuccini F, Niazman M, et al. Thorax irradiation triggers a local and systemic accumulation of immunosuppressive CD4+ FoxP3+ regulatory T cells. Radiat Oncol 2014;9:98.

17. Cappuccini F, Eldh T, Bruder D, et al. New insights into the molecular pathology of radiation-induced pneumopathy. Radiother Oncol 2011;101:86-92.

18. Paun A, Kunwar A, Haston CK. Acute adaptive immune response correlates with late radiation-induced pulmonary fibrosis in mice. Radiat Oncol 2015;10:45.

19. Wynn TA. Fibrotic disease and the $\mathrm{T}(\mathrm{H}) 1 / \mathrm{T}(\mathrm{H}) 2$ paradigm. Nat Rev Immunol 2004;4:583-94.

20. Huehn J, Siegmund K, Lehmann JC, et al. Developmental stage, phenotype, and migration distinguish naive- and effector/memory-like CD4+ regulatory T cells. J Exp Med 2004;199:303-13.

21. Xiong S, Pan X, Xu L, et al. Regulatory T Cells Promote $\beta$-Catenin--Mediated Epithelium-to-Mesenchyme Transition During Radiation-Induced Pulmonary Fibrosis. Int J Radiat Oncol Biol Phys 2015;93:425-35.

22. Xiong $S$, Guo R, Yang $Z$, et al. Treg depletion attenuates irradiation-induced pulmonary fibrosis by reducing fibrocyte accumulation, inducing Th17 response, and shifting IFN- $\gamma$, IL-12/IL-4, IL-5 balance. Immunobiology
2015;220:1284-91.

23. Sheng Y, Chen K, Jiang W, et al. PD-1 restrains IL17A production from $\gamma \delta \mathrm{T}$ cells to modulate acute radiation-induced lung injury. Transl Lung Cancer Res 2021;10:685-98.

24. Gordon S, Martinez FO. Alternative activation of macrophages: mechanism and functions. Immunity 2010;32:593-604.

25. Li M, Liu P, Ke Y, et al. Research progress on macrophage in radiation induced lung injury. Zhejiang Da Xue Xue Bao Yi Xue Ban 2020;49:623-8.

26. S N SG, Raviraj R, Nagarajan D, et al. Radiation-induced lung injury: impact on macrophage dysregulation and lipid alteration - a review. Immunopharmacol Immunotoxicol 2019;41:370-9.

27. Araya J, Nishimura SL. Fibrogenic reactions in lung disease. Annu Rev Pathol 2010;5:77-98.

28. Jin H, Yoo Y, Kim Y, et al. Radiation-Induced Lung Fibrosis: Preclinical Animal Models and Therapeutic Strategies. Cancers (Basel) 2020;12:1561.

29. Ikushima H, Miyazono K. TGFbeta signalling: a complex web in cancer progression. Nat Rev Cancer 2010;10:415-24.

30. Ho IC, Miaw SC. Regulation of IL-4 Expression in Immunity and Diseases. Adv Exp Med Biol 2016;941:31-77.

31. Mosser DM, Edwards JP. Exploring the full spectrum of macrophage activation. Nat Rev Immunol 2008;8:958-69.

32. Jakubzick C, Kunkel SL, Puri RK, et al. Therapeutic targeting of IL-4- and IL-13-responsive cells in pulmonary fibrosis. Immunol Res 2004;30:339-49.

33. Lee JW, Zoumalan RA, Valenzuela CD, et al. Regulators and mediators of radiation-induced fibrosis: Gene expression profiles and a rationale for $\mathrm{Smad} 3$ inhibition. Otolaryngol Head Neck Surg 2010;143:525-30.

34. Gurujeyalakshmi G, Giri SN. Molecular mechanisms of antifibrotic effect of interferon gamma in bleomycinmouse model of lung fibrosis: downregulation of TGFbeta and procollagen I and III gene expression. Exp Lung Res 1995;21:791-808.

35. Ulloa L, Doody J, Massagué J. Inhibition of transforming growth factor-beta/SMAD signalling by the interferongamma/STAT pathway. Nature 1999;397:710-3.

36. Penke LR, Speth JM, Dommeti VL, et al. FOXM1 is a critical driver of lung fibroblast activation and fibrogenesis. J Clin Invest 2018;128:2389-405.

37. Huang SK, Wettlaufer SH, Chung J, et al. Prostaglandin $\mathrm{E} 2$ inhibits specific lung fibroblast functions via selective actions of PKA and Epac-1. Am J Respir Cell Mol Biol 
2008;39:482-9.

38. Thomas PE, Peters-Golden M, White ES, et al. PGE(2) inhibition of TGF-beta1-induced myofibroblast differentiation is Smad-independent but involves cell shape and adhesion-dependent signaling. Am J Physiol Lung Cell Mol Physiol 2007;293:L417-28.

39. Boyle JE, Lindroos PM, Rice AB, et al. Prostaglandin-E2 counteracts interleukin-1beta-stimulated upregulation of platelet-derived growth factor alpha-receptor on rat pulmonary myofibroblasts. Am J Respir Cell Mol Biol 1999;20:433-40.

40. Bozyk PD, Moore BB. Prostaglandin E2 and the pathogenesis of pulmonary fibrosis. Am J Respir Cell Mol Biol 2011;45:445-52.

41. Rodrigues G, Lock M, D'Souza D, et al. Prediction of radiation pneumonitis by dose - volume histogram parameters in lung cancer--a systematic review. Radiother Oncol 2004;71:127-38.

42. Choi YW, Munden RF, Erasmus JJ, et al. Effects of radiation therapy on the lung: radiologic appearances and differential diagnosis. Radiographics 2004;24:985-97; discussion 998.

43. Jain A, Shannon VR, Sheshadri A. Immune-Related Adverse Events: Pneumonitis. Adv Exp Med Biol 2018;995:131-49.

44. Graves PR, Siddiqui F, Anscher MS, et al. Radiation pulmonary toxicity: from mechanisms to management. Semin Radiat Oncol 2010;20:201-7.

45. Ullah T, Patel H, Pena GM, et al. A contemporary review of radiation pneumonitis. Curr Opin Pulm Med 2020;26:321-5.

46. Barnes PJ. Glucocorticosteroids: current and future directions. Br J Pharmacol 2011;163:29-43.

47. Adcock IM, Mumby S. Glucocorticoids. Handb Exp Pharmacol 2017;237:171-96.

48. Starick SR, Ibn-Salem J, Jurk M, et al. ChIP-exo signal associated with DNA-binding motifs provides insight into the genomic binding of the glucocorticoid receptor and cooperating transcription factors. Genome Res 2015;25:825-35.

49. Vandewalle J, Luypaert A, De Bosscher K, et al. Therapeutic Mechanisms of Glucocorticoids. Trends Endocrinol Metab 2018;29:42-54.

50. Mattishent K, Thavarajah M, Blanco P, et al. Meta-review: adverse effects of inhaled corticosteroids relevant to older patients. Drugs 2014;74:539-47.

51. Zhang C, Zhu Y, Zhang Y, et al. Therapeutic Potential of Umbilical Cord Mesenchymal Stem Cells for Inhibiting Myofibroblastic Differentiation of Irradiated Human Lung Fibroblasts. Tohoku J Exp Med 2015;236:209-17.

52. Dong LH, Jiang YY, Liu YJ, et al. The anti-fibrotic effects of mesenchymal stem cells on irradiated lungs via stimulating endogenous secretion of HGF and PGE2. Sci Rep 2015;5:8713.

53. D'Hellencourt CL, Diaw L, Cornillet P, et al. Differential regulation of TNF alpha, IL-1 beta, IL-6, IL-8, TNF beta, and IL-10 by pentoxifylline. Int J Immunopharmacol 1996;18:739-48.

54. Rocha-Santos V, Figueira ER, Rocha-Filho JA, et al. Pentoxifylline enhances the protective effects of hypertonic saline solution on liver ischemia reperfusion injury through inhibition of oxidative stress. Hepatobiliary Pancreat Dis Int 2015;14:194-200.

55. Ozturk B, Egehan I, Atavci S, et al. Pentoxifylline in prevention of radiation-induced lung toxicity in patients with breast and lung cancer: a double-blind randomized trial. Int J Radiat Oncol Biol Phys 2004;58:213-9.

56. Delanian S, Porcher R, Rudant J, et al. Kinetics of response to long-term treatment combining pentoxifylline and tocopherol in patients with superficial radiationinduced fibrosis. J Clin Oncol 2005;23:8570-9.

57. Delanian S, Porcher R, Balla-Mekias S, et al. Randomized, placebo-controlled trial of combined pentoxifylline and tocopherol for regression of superficial radiation-induced fibrosis. J Clin Oncol 2003;21:2545-50.

58. Kuo CH, Lee MS, Kuo HF, et al. Azithromycin suppresses Th1- and Th2-related chemokines IP-10/MDC in human monocytic cell line. J Microbiol Immunol Infect 2019;52:872-9.

59. Tang F, Li R, Xue J, et al. Azithromycin attenuates acute radiation-induced lung injury in mice. Oncol Lett 2017;14:5211-20.

60. Molthen RC, Wu Q, Fish BL, et al. Mitigation of radiation induced pulmonary vascular injury by delayed treatment with captopril. Respirology 2012;17:1261-8.

61. Medhora M, Gao F, Jacobs ER, et al. Radiation damage to the lung: mitigation by angiotensin-converting enzyme (ACE) inhibitors. Respirology 2012;17:66-71.

62. Kharofa J, Cohen EP, Tomic R, et al. Decreased risk of radiation pneumonitis with incidental concurrent use of angiotensin-converting enzyme inhibitors and thoracic radiation therapy. Int J Radiat Oncol Biol Phys 2012;84:238-43. 
63. Choi SB, Bae GS, Jo IJ, et al. Effects of Berberine on Acute Necrotizing Pancreatitis and Associated Lung Injury. Pancreas 2017;46:1046-55.

64. Vujaskovic Z, Feng QF, Rabbani ZN, et al. Assessment of the protective effect of amifostine on radiation-induced pulmonary toxicity. Exp Lung Res 2002;28:577-90.

65. Xie D, Chen A, Lv S, et al. Late-course accelerated hyperfractionated intensity-modulated radiotherapy for nasopharyngeal adenoid cystic carcinoma: A case report. Med Dosim 2020;45:46-51.

66. Shinde A, Li R, Kim J, et al. Stereotactic body radiation therapy (SBRT) for early-stage lung cancer in the elderly. Semin Oncol 2018;45:210-9.

67. Tsujino K, Hirota S, Endo M, et al. Predictive value of dose-volume histogram parameters for predicting radiation pneumonitis after concurrent chemoradiation for lung cancer. Int J Radiat Oncol Biol Phys 2003;55:110-5.

68. Tsujino K, Hashimoto T, Shimada T, et al. Combined analysis of V20, VS5, pulmonary fibrosis score on baseline computed tomography, and patient age improves prediction of severe radiation pneumonitis after concurrent chemoradiotherapy for locally advanced non-small-cell lung cancer. J Thorac Oncol 2014;9:983-90.

69. Marks LB, Bentzen SM, Deasy JO, et al. Radiation dosevolume effects in the lung. Int J Radiat Oncol Biol Phys 2010;76:S70-6.

70. Fournel P, Robinet G, Thomas P, et al. Randomized phase III trial of sequential chemoradiotherapy compared with concurrent chemoradiotherapy in locally advanced non-small-cell lung cancer: Groupe Lyon-SaintEtienne d'Oncologie Thoracique-Groupe Français de Pneumo-Cancérologie NPC 95-01 Study. J Clin Oncol 2005;23:5910-7.

71. Vogelius IR, Bentzen SM. A literature-based meta-analysis of clinical risk factors for development of radiation induced pneumonitis. Acta Oncol 2012;51:975-83.

72. Hernandez C, Huebener P, Schwabe RF. Damageassociated molecular patterns in cancer: a double-edged sword. Oncogene 2016;35:5931-41.

73. Gubin MM, Zhang X, Schuster H, et al. Checkpoint blockade cancer immunotherapy targets tumour-specific mutant antigens. Nature 2014;515:577-81.

74. Wirsdörfer F, de Leve S, Jendrossek V. Combining Radiotherapy and Immunotherapy in Lung Cancer: Can We Expect Limitations Due to Altered Normal Tissue
Toxicity? Int J Mol Sci 2018;20:24.

75. Shaverdian N, Lisberg AE, Bornazyan K, et al. Previous radiotherapy and the clinical activity and toxicity of pembrolizumab in the treatment of non-small-cell lung cancer: a secondary analysis of the KEYNOTE-001 phase 1 trial. Lancet Oncol 2017;18:895-903.

76. Antonia SJ, Villegas A, Daniel D, et al. Durvalumab after Chemoradiotherapy in Stage III Non-Small-Cell Lung Cancer. N Engl J Med 2017;377:1919-29.

77. Li YL, Zhao H, Ren XB. Relationship of VEGF/VEGFR with immune and cancer cells: staggering or forward? Cancer Biol Med 2016;13:206-14.

78. Qi WX, Sun YJ, Shen Z, et al. Risk of interstitial lung disease associated with EGFR-TKIs in advanced nonsmall-cell lung cancer: a meta-analysis of 24 phase III clinical trials. J Chemother 2015;27:40-51.

79. Albain KS, Swann RS, Rusch VW, et al. Radiotherapy plus chemotherapy with or without surgical resection for stage III non-small-cell lung cancer: a phase III randomised controlled trial. Lancet 2009;374:379-86.

80. Moreno M, Aristu J, Ramos LI, et al. Predictive factors for radiation-induced pulmonary toxicity after threedimensional conformal chemoradiation in locally advanced non-small-cell lung cancer. Clin Transl Oncol 2007;9:596-602.

81. Monson JM, Stark P, Reilly JJ, et al. Clinical radiation pneumonitis and radiographic changes after thoracic radiation therapy for lung carcinoma. Cancer 1998;82:842-50.

82. Rancati T, Ceresoli GL, Gagliardi G, et al. Factors predicting radiation pneumonitis in lung cancer patients: a retrospective study. Radiother Oncol 2003;67:275-83.

83. Robnett TJ, Machtay M, Vines EF, et al. Factors predicting severe radiation pneumonitis in patients receiving definitive chemoradiation for lung cancer. Int $\mathrm{J}$ Radiat Oncol Biol Phys 2000;48:89-94.

84. Nakamura T, Fuwa N, Kodaira T, et al. Clinical outcome of stage III non-small-cell lung cancer patients after definitive radiotherapy. Lung 2008;186:91-6.

85. Fay M, Tan A, Fisher R, et al. Dose-volume histogram analysis as predictor of radiation pneumonitis in primary lung cancer patients treated with radiotherapy. Int J Radiat Oncol Biol Phys 2005;61:1355-63.

86. Wang S, Liao Z, Wei X, et al. Association between systemic chemotherapy before chemoradiation and increased risk of treatment-related pneumonitis in 
esophageal cancer patients treated with definitive chemoradiotherapy. J Thorac Oncol 2008;3:277-82.

87. Claude L, Pérol D, Ginestet C, et al. A prospective study on radiation pneumonitis following conformal radiation therapy in non-small-cell lung cancer: clinical and dosimetric factors analysis. Radiother Oncol 2004;71:175-81.

(English Language Editor: A. Kassem)

Cite this article as: Yan Y, Fu J, Kowalchuk RO, Wright CM, Zhang R, Li X, Xu Y. Exploration of radiation-induced lung injury, from mechanism to treatment: a narrative review. Transl Lung Cancer Res 2022;11(2):307-322. doi: 10.21037/tlcr-22108 